\title{
Du rural aux nouvelles ruralités
}

From rural to new rurality

De lo rural a las nuevas ruralidades

\section{Laurent Rieutort}

\section{OpenEdition}

\section{Journals}

Édition électronique

URL : http://journals.openedition.org/ries/2267

DOI : $10.4000 /$ ries.2267

ISSN : 2261-4265

Éditeur

Centre international d'études pédagogiques

Édition imprimée

Date de publication : 1 avril 2012

Pagination : 43-52

ISBN : 978-2-85420-594-7

ISSN : 1254-4590

\section{Référence électronique}

Laurent Rieutort, «Du rural aux nouvelles ruralités », Revue internationale d'éducation de Sèvres [En ligne], 59 | avril 2012, mis en ligne le 06 février 2015, consulté le 19 avril 2019. URL : http:// journals.openedition.org/ries/2267 ; DOI : 10.4000/ries.2267 


\section{Du rural aux nouvelles ruralités}

\section{Laurent Rieutort}

La ruralité désigne l'ensemble de représentations collectives et de caractères concourant à une forme d'identité et de fonctionnement des espaces ruraux. Sa définition exige donc de revenir sur la question du " rural » et de s'inscrire, de plus en plus, dans la dialectique des rapports ville-campagne, avec des modifications récentes dans les perceptions, les pratiques et les modalités de gouvernance de ces espaces. L'ensemble des processus conduit à revisiter la question de l'éducation dans les territoires ruraux.

\section{LE RURAL, DU LOCAL AU GLOBAL}

La définition de l'espace "rural » demeure délicate, alimentant des débats récurrents entre chercheurs qui refusent de perpétuer une définition par la négative (tout ce qui n'est pas urbain... est rural). Comme le note N. Mathieu ${ }^{1}$, "d'absorption en périurbanisation, la distinction s'efface » pour « deux entités que la tradition veut distinctes, mais que les évolutions sociales, économiques, professionnelles récentes tendent à rapprocher $»^{2}$. Prudent, le dictionnaire de la géographie et de l'espace des sociétés (Lévy, Lussault, 2003) considère que le rural «désigne globalement les campagnes dans leur complexité sans réduire celles-ci aux manifestations des activités agricoles ». Pour le tropicaliste R. Pourtier, le territoire rural «ne se caractérise pas seulement par les particularités bio-physiques de l'étendue qui lui sert de support, les techniques et les modes de production des populations qui l'exploitent et les droits d'usage exclusifs qu'elles revendiquent ; il se caractérise aussi par son degré d'intégration économique et sociale dans un espace chaque jour davantage réticulaire, et par la force des sentiments identitaires et d'appropriation que les habitants nourrissent à son égard $»^{3}$.

Dans la définition classique "dichotomique " se pose alors la question de la délimitation de la ville, que celle-ci s'inscrive sur une vision purement statistique, n'impliquant rien de particulier dans le domaine de la gestion des territoires, ou qu'elle se fonde sur de stricts critères politico-administratifs (l'État décide d'attribuer le "statut » de ville à une localité) et donc sur des différences de fonctionnement ou de traitement.

1. Les références des articles cités figurent en note de bas de page lors de leur première occurrence. Les occurrences suivantes renvoient à la note initiale. Les ouvrages cités figurent dans la bibliographie située en fin d'article ( $N d I R)$.

2. Mathieu N., 2004, "Relations ville-campagne : quel sens, quelle évolution ? ", Revue Pour, n 182.

3. Pourtier, R., 2004, "Développement "rurable" au Sud, géodiversité et dynamiques territoriales », Historiens et Géographes, $\mathrm{n}^{\circ} 187$, p. 217-230. 
La conception statistique varie beaucoup d'un pays à l'autre. En France, rappelons que depuis $1853^{4}$, appartiennent à la campagne les communes de moins de deux mille habitants agglomérés ${ }^{5}$. Dans d'autres pays du monde, ce seuil de population, parfois croisé avec des données sur les densités, varie généralement entre mille et cinq mille, mais il peut s'abaisser à deux cents ou trois cents (Danemark, Islande) ou, au contraire monter à dix mille (Espagne) ou même cinquante mille (Japon). Les Nations Unies, qui ont opté pour le seuil des vingt mille habitants, considèrent qu'en 2010, $49 \%$ de la population mondiale résident à la campagne, soit plus de 3,3 milliards de personnes. Les citadins sont devenus majoritaires en 2008, alors que seulement un tiers de la population mondiale vivait dans les zones urbaines en 1960 et encore $47 \%$ en 2000. On devrait atteindre $60 \%$ en 2030 mais le nombre de ruraux augmente encore en valeur absolue (environ $+1,6 \%$ par an entre 1960 et $1980,+1 \%$ par an entre 1980 et 2000 et même $+1,1 \%$ par an depuis l'an 2000). Il faut dire que dans les pays du Sud, les campagnes sont plus «fécondes » que les villes. La natalité restant stable du fait des structures par âge, la population augmente encore, à l'exception de l'Asie orientale. Les enfants, moins scolarisés, restent plus longtemps dans les familles et dans des campagnes où ils travaillent très jeunes. Ainsi, les zones rurales demeurent des réservoirs de croissance démographique, même si elles alimentent, via l'émigration, les métropoles et villes intermédiaires.

Dans ces conditions, on comprend que les pays à revenus faible et intermédiaire concentrent $92 \%$ de la population rurale, les pays les moins avancés près de $20 \%$. On devine le poids de l'Afrique sud-saharienne (avec 534 millions de campagnards) et surtout de l'Asie méridionale et orientale (avec 2,2 milliards). Cela représente environ $60 \%$ de la population de ces deux continents qui vit à la campagne. Dans les pays développés, profitant d'une nouvelle attractivité migratoire, le nombre de ruraux s'est stabilisé (il a même augmenté en Amérique du Nord lors du «rebond» des années 1970-1980) même si sa part dans la population continue à décroître ( $26 \%$ dans l'Union européenne, $18 \%$ en Amérique du Nord).

À côté des statistiques démographiques, d'autres approches quantitatives ou plus qualitatives peuvent reposer sur des données économiques (poids de l'agriculture et de l'exploitation forestière), sociales (taille des " communautés ", faible mobilité, interdépendance entre les individus, fort contrôle social et maîtrise politique de la mobilité) ou paysagères (motifs agro-sylvopastoraux). Parallèlement, les chercheurs montrent les écarts de développement ou d'équipements entre espaces ruraux et urbains. C'est ainsi que l'accès aux services de santé et d'éducation est souvent plus difficile dans les campagnes,

4. Kayser B., 1988, « Permanence et perversion de la ruralité », Études rurales, 109, p. 75-108.

5. Cette notion d'agglomération, donc de densité du bâti, correspond à la règle des moins de deux cent mètres entre les constructions dans les unités urbaines. 


\begin{tabular}{|l|r|r|r|r|r|c|}
\cline { 2 - 7 } \multicolumn{1}{c|}{} & \multicolumn{5}{c|}{ Population rurale (en valeur absolue et en millions) } \\
\cline { 2 - 8 } & 1960 & 1970 & 1980 & 1990 & 2000 & 2010 \\
\hline Monde & 2026 & 2347 & 2686 & 2994 & 3227 & 3349 \\
\hline Asie de l'Est et Pacifique & 822 & 979 & 1135 & 1201 & 1198 & 1114 \\
\hline Asie du Sud & 467 & 578 & 694 & 842 & 988 & 1103 \\
\hline Europe et Asie centrale & 299 & 290 & 275 & 268 & 268 & 266 \\
\hline Union européenne & 157 & 150 & 138 & 134 & 132 & 130 \\
\hline Amérique latine et Caraïbes & 112 & 122 & 126 & 129 & 128 & 122 \\
\hline Afrique du Nord et Moyen-Orient & 68 & 79 & 93 & 114 & 129 & 146 \\
\hline Afrique subsaharienne & 196 & 237 & 295 & 368 & 448 & 534 \\
\hline Amérique du Nord & 59 & 59 & 65 & 68 & 65 & 61 \\
\hline
\end{tabular}

\begin{tabular}{|l|c|c|c|c|c|c|}
\cline { 2 - 7 } \multicolumn{2}{c|}{} & \multicolumn{7}{c|}{ Population rurale (en \%) } \\
\cline { 2 - 8 } & $\mathbf{1 9 6 0}$ & $\mathbf{1 9 7 0}$ & $\mathbf{1 9 8 0}$ & $\mathbf{1 9 9 0}$ & $\mathbf{2 0 0 0}$ & $\mathbf{2 0 1 0}$ \\
\hline Monde & $\mathbf{6 7}$ & $\mathbf{6 4}$ & $\mathbf{6 1}$ & $\mathbf{5 7}$ & $\mathbf{5 3}$ & $\mathbf{4 9}$ \\
\hline Asie de l'Est et Pacifique & 79 & 77 & 74 & 67 & 59 & 52 \\
\hline Asie du Sud & 83 & 81 & 78 & 75 & 73 & 70 \\
\hline Europe et Asie centrale & 45 & 39 & 35 & 32 & 31 & 30 \\
\hline Union européenne & 39 & 34 & 30 & 28 & 27 & 26 \\
\hline Amérique latine et Caraïbes & 51 & 43 & 35 & 29 & 25 & 21 \\
\hline Afrique du Nord et Moyen-Orient & 65 & 57 & 50 & 45 & 41 & 38 \\
\hline Afrique subsaharienne & 85 & 80 & 76 & 72 & 67 & 62 \\
\hline Amérique du Nord & 30 & 26 & 26 & 24 & 21 & 18 \\
\hline
\end{tabular}

Source : ONU. La population rurale fait référence aux personnes vivant en milieu rural tel que défini par les bureaux nationaux de statistiques.

que celles-ci enregistrent encore de fortes densités de population ou qu'elles soient marquées par une déprise humaine. En matière de soins comme d'école, l'accessibilité pose de sérieux problèmes d'aménagement, notamment parce qu'il convient d'envisager les différentes dimensions du concept ${ }^{6}$.

6. Le concept comprend la disponibilité (availability) par rapport aux besoins de la population, l'accessibilité (accessibility) qui met en relation la localisation de l'offre et celle de la population, en prenant en compte les moyens de transport, le temps et la distance de trajet voire le coût, l'hébergement (accomodation), manière dont est organisée l'offre et la perception qu'en ont les usagers, l'abordabilité (affordability) introduisant la perception de l'individu sur la valeur du coût total du service, et enfin l'acceptabilité (acceptability) qui renvoie aux préoccupations sociales et culturelles. Voir : Penchansky R., Thomas W., 1981, « The Concept of Access: Definition and Relationship to Consumer Satisfaction ", Medical Care, 19, p. 127-140. 
Pour ne prendre que l'exemple des montagnes isolées de HauteProvence, on constate une complexification des enjeux entre les zones très peu denses et vieillies, où se pose la question du maintien des écoles, voire des collèges, et des zones - parfois très proches - bénéficiant de la nouvelle attractivité migratoire (souvent des gains démographiques de 1 à $2 \%$ par an) qui entraine une formidable diversification de la société locale (ménages aisés dont les enfants ne fréquentent pas toujours l'école rurale, familles en difficulté, catégories sociales "moyennes" accédant à la propriété dans un cadre "rural " désiré)... et des attentes en matière d'éducation. L'exigence de qualité ou de diversité de l'offre est de plus en plus grande, mettant en concurrence les pôles ruraux et urbains. En outre, les nouvelles populations ont parfois du mal à s'intégrer dans des campagnes qui offrent peu d'emplois, où les municipalités ont peu de moyens, et où les propositions de logements sont somme toute réduites. Il s'ensuit un turn over particulièrement important, avec des conséquences au niveau du suivi des élèves, le renouvellement des effectifs pouvant atteindre $30 \%$ d'une année sur l'autre ${ }^{7}$.

En définitive, les recompositions socio-spatiales modifient la donne "rurale ». Dans les pays anciennement industrialisés, le bâti de type "urbain » se diffuse dans des campagnes "mitées " par la "rurbanisation », les "ruraux » adoptent les mêmes modes de vie, les mêmes mobilités et représentations que les citadins, tandis que les catégories socioprofessionnelles se diversifient, conduisant à la marginalisation des agriculteurs; désormais le rural n'est plus l'agricole et l'on passe d'une société d'interconnaissance à un système plus ouvert et plus éclaté (dissociation des lieux de résidence et d'emploi, résidences alternantes).

Dans les pays du Sud, ces mêmes bouleversements sont en cours: amélioration des moyens de communication et ouverture, mobilités multiples faites d'allers et retours, diversification de l'emploi avec la progression de la pluriactivité, de l'agriculture commerciale (le "vivrier-marchand»), des activités artisanales, industrielles et tertiaires (notamment touristiques), souvent grâce à la vigueur des réseaux animés par les villes. Les nouveaux revenus tirés de ces activités permettent de s'équiper mais aussi de développer des stratégies de scolarisation des enfants en ville. Dans de nombreux cas, la dégradation de la conjoncture économique a ajouté aux «migrants de retour » classiques (héritiers, retraités, ...) de nouveaux types d'émigrants urbains (chômeurs, jeunes déscolarisés, etc.). Parfois confrontés à des difficultés d'insertion, ces derniers contribuent à la création de nouvelles activités, transformant l'économie et l'espace des villages dans lesquels ils s'installent. Comme dans les pays occidentaux, la campagne proche des grandes agglomérations devient même un espace résidentiel en raison des coûts du logement et de meilleures liaisons routières.

7. Source : enquêtes personnelles 2011, Pays « Asses, Verdon, Vaïre, Var », canton de Barrême. 
En Chine, on assiste depuis peu à un mouvement de revalorisation des zones rurales, profitant de séjours touristiques et d'une réorientation des activités locales (artisanat, parcs agricoles) (Sanjuan in Guibert, Jean, 2011).

On peut alors aboutir à une remise en cause de la notion même de « campagne » en argumentant sur un «continuum» entre ville et monde rural. En réalité, les termes du débat sont mal posés comme le souligne B. Kayser : «le "tout-est-urbain" repose sur une confusion entre mode de vie et pratique de consommation et une équivoque sur le sens du mot urbanisation "; on doit alors « rejeter la domination des références : qu'elles soient socio-spatiales - le rural par rapport à l'urbain -, ou temporelles - le rural d'aujourd'hui par rapport à son passé » (voir note 4). Les équations sont souvent réductrices : l'espace rural n'est pas uniquement agricole et la campagne est loin d'être un environnement aussi naturel qu'il y paraît aux yeux des citadins. En outre, les définitions se heurtent à la diversité géographique des espaces concernés, aux différents contextes socio-économiques ou aux gradients d'influence des cités, depuis les «campagnes des villes » en position périurbaine jusqu'aux milieux ruraux plus isolés qui n'ignorent pas pour autant l'influence urbaine. B. Jean (2002) insiste d'ailleurs sur « un accroissement des différenciations internes de la ruralité elle-même, plus significatif pour comprendre la ruralité dans la modernité que l'épineux exercice d'opposer en bloc le rural à l'urbain ». Cette invitation à revoir les typologies des espaces ruraux est d'ailleurs suivie de multiples recherches (Bonnamour, 1993).

Au final, les chercheurs privilégient désormais un critère "géographique » lié à la relative faible densité, non seulement démographique, " mais également de constructions, d'emplois, d'équipements, de commerces, de services, de voies de communications et, plus généralement d'interconnections ${ }^{8}$ (R. Chapuis). Il en résulte une vision moins thématique, plus globale, l'espace rural en recomposition s'identifiant par un mode spécifique d'utilisation du sol voire de vie sociale et de représentations encore fortement connotées par l'agriculture ou des valeurs environnementales et patrimoniales. Ces définitions exigent toutefois de ne pas considérer les campagnes ou les villes comme des isolats, des mondes clos, mais plutôt comme des espaces en interaction et dont les limites s'effacent, au sein d'un même "système " global qui contribue à les structurer et à les modifier. Au Sud, les campagnes, y compris les plus isolées, "participent indéniablement au grand mouvement mondial qui se traduit par une déconnexion croissante entre agriculture et monde rural ; elles le font néanmoins de façon spécifique, dans des contextes géographiques fortement marqués par l'héritage colonial, le poids des dépendances à l'égard d'acteurs extérieurs, les spécificités des milieux naturels, des dynamiques démographiques propres. La géographie des campagnes tropicales est de moins en moins assimilable à la

8. Chapuis R., « Espace rural », Encyclopédie en ligne Hypergéo. 
géographie des seuls systèmes et structures agraires et relève de plus en plus d'une approche d'une complexité multiscalaire où les logiques locales cohabitent avec les logiques globales » ${ }^{9}$.

\section{LA RURALITÉ, DES FAITS AUX REPRÉSENTATIONS}

Classiquement, le terme de ruralité indiquait : 1) le caractère de ce qui est « rural », les caractéristiques des espaces ruraux ; 2) l' " ensemble des valeurs, de la culture propre au milieu rural » ou à la " condition des campagnards » (Littré, 1880) ; par opposition à l'« urbanité » qui désignait les traits culturels positifs ("civilité », usages policés, courtoisie, etc.) que l’on assurait être spécifiques aux citadins, la "ruralité » pouvait ainsi renvoyer de façon péjorative à la "rusticité ", au manque de savoir-vivre de ses habitants, au caractère "périphérique ». À partir des années 1970, de nouvelles catégories du discours et de l'action ont contribué à réinterroger la ruralité. Ainsi, dans les pays occidentaux, une idéologie anti-ville est apparue et « on en revient, a contrario, à une définition valorisante de la ruralité ${ }^{10}$. La ruralité devient un idéal (liberté, épanouissement, équilibre). Face aux enjeux d'aménagement, elle prend même une dimension politique, comme en France, où on finira par créer un ministère de l'agriculture, de l'alimentation, de la pêche et de la ruralité en 2004, avant d'instituer une «Conférence de la ruralité » avec la loi du 23 février 2005 relative au développement des territoires ruraux.

Pour autant, la dimension idéologique du terme et notamment la tendance à idéaliser la vie à la campagne ( "ruralisme »), entraîne une méfiance des chercheurs qui continuent à préférer les termes de "campagne » ou de « rural », au moment où d'ailleurs se développent dans les pays occidentaux des débats épistémologiques sur l'existence d'une société - voire d'une réalité spécifiquement rurale. Comment penser l'appartenance locale, l'identité « rurale » dans ce contexte de mobilité accentuée ? Dans les années 1980, des recherches dans les pays occidentaux avaient pourtant montré un processus de « retour au local», de "relocalisation » de la vie sociale, avec une nouvelle attractivité migratoire des campagnes qui ouvrait la voie à la thèse d'une "renaissance rurale " (Kayser, 1989) ; le rural étant redécouvert, " choisi », car il permet de retrouver une sociabilité de proximité et d'interconnaissance à l'opposé d'un supposé anonymat urbain. Vers 1990, de nouvelles lectures de la ruralité sont proposées. Des auteurs considèrent que l'espace rural conserve certaines spécificités par les représentations qu'il suscite et notamment grâce aux rapports particuliers qu'il entretient avec l'environnement et la «nature». Le rural

9. Bart F., 2010, « Dynamiques des campagnes tropicales : introduction », Les Cahiers d'Outre-Mer, 249, p. 3-6. 10. Mathieu N., 1998, "La notion de rural et les rapports ville/campagne en France : les années quatre-vingt-dix », Économie rurale, $\mathrm{n}^{\circ} 247$, p. 11-20. 
devient alors un objet à protéger ou à mettre en valeur et qui touche toute la société "globale». Cet espace assure des fonctions et des usages multiples qui entraînent concurrences et conflits mais aussi complémentarités voire «multifonctionnalité » que les acteurs du développement appellent de leurs vœux. Pour B. Kayser, «la ruralité n'est ni le contraire de l'urbanité, ni son prolongement, ni la dégradation de son état ancien, ni sa résurgence " mais bien une forme de rapport à l'espace, une inscription dans le "local », voire une "familiarité des lieux» (voir note 4) ; les sentiments d'appartenance au rural pouvant dans les pays occidentaux découler d'un choix de vie, d'un imaginaire positif (valeurs symboliques à l'origine des récentes migrations d'agrément par exemple), voire d'un arbitrage économique.

D'autres chercheurs « constructivistes » contestent l'hypothèse d'une superposition entre espace rural et société rurale. B. Hervieu et J. Viard (1996), différencient ainsi les catégories de sens - l'urbanité et la ruralité - des réalités géographiques - la ville et la campagne. Y. Sancébé distingue l'espace géographique (la campagne et ses paysages), l'espace social (habitants, usagers, acteurs du rural) et l'espace symbolique (valeurs, fonctions attribuées à la ruralité). C'est ainsi que l'opposition urbain/rural persiste dans les imaginaires, notamment des pays occidentaux : "la représentation d'une différence entre "urbanité » et "ruralité » d'un mode de vie demeure ancrée dans les esprits de tous ceux qui cherchent par leur mobilité et migration à en associer les avantages ${ }^{11}$. En d'autres termes, l'urbanité est devenue un opérateur du fonctionnement et de l'organisation de l'espace rural ; la société urbaine "globale » porte un droit de regard et d'usage sur ces espaces devenus "publics». Des acteurs multiples réinvestissent la campagne de valeurs symboliques et de fonctions spécifiques (entretien de l'espace, productions de "terroir » et d'aliments de qualité, qualité de vie résidentielle). Se faisant, on rapproche des modes de vie citadins et des valeurs perçues comme rurales (appartenance locale, convivialité, temps pour soi...), et l'on peut même observer « une certaine ruralisation de fragments d'espaces et de sociétés urbaines » (M. Vanier in Arlaud et al., 2005).

\section{LES NOUVELLES RURALITÉS : DU RURAL À L'URBAIN}

Dans les nouvelles ruralités, villes et campagnes sont donc étroitement liées, même si évidemment des écarts demeurent en termes de conditions de vie et d'accès aux services par exemple. Mais, le rural, l'urbain, les territoires locaux et les espaces nationaux voire transnationaux, sont étroitement imbriqués et interdépendants; ils sont solidairement entrés dans l'ère de la globalisation et de la compétition. Rappelons que la question des rapports entre ville et campagne 
est une préoccupation ancienne dans les pays dits développés ou en Amérique latine, alors qu'elle suscite jusque dans les années 1980 une certaine indifférence en Afrique ${ }^{12}$. À partir des années 1970, le thème des relations ville/campagne est renouvelé : à l'opposition classique se superpose désormais une homogénéisation des espaces. La ville répand inexorablement sa culture, ses modes de vie, ses produits de consommation, ses pratiques... et son bâti résidentiel. On parle d'annexion et "d'urbanisation des campagnes " ${ }^{13}$; le rural est au mieux « interstitiel ", " résiduel », face à une modernisation "urbaine » qui " consomme » l'espace. Si d'aucuns soulignent des gradients socio-économiques ou démographiques entre la ville et des campagnes dites "profondes », les déterminants sont clairement urbains. On devine les présupposés idéologiques sous-jacents : le progrès et la modernisation sont en ville tandis que le rural est symbole d'archaïsme, de retard de développement, de handicaps multiples... B. Kayser (voir note 4) ou N. Mathieu ${ }^{14}$ décèlent surtout la montée en puissance d'un modèle reposant sur une hiérarchie entre les valeurs des lieux (qualité de vie, ressources naturelles et patrimoniales) à l'avantage de la ruralité ; les nouvelles polarités migratoires dessinant une nouvelle relation à la ville et construisant "sur des bases démographiques et résidentielles, des territoires qui ne sont que des "espaces" produits par ces migrations" (voir note 1).

Face à la diversification des usages, aux nouvelles articulations entre lieux de résidences et de travail, aux appartenances multiples, on doit donc désormais raisonner en termes de "dialogue rural/urbain » et non de façon dualiste, même si subsistent des conceptions antagonistes ${ }^{15}$. Dans les pays en développement, les chercheurs remettent également en cause la coupure villecampagne, en montrant la pénétration des influences urbaines dans le monde rural (la ville est alors créatrice de campagnes) et parfois la persistance du rural dans la ville (dans les pratiques agricoles comme dans les constructions sociales). Le renforcement des solidarités tire profit de l'amélioration des modes de transport et de circulation des capitaux, des réseaux familiaux ou villageois et d'activités marchandes jouant sur les proximités urbaines. L'agriculture vivrière à destination des villes est devenue une activité centrale, souvent plus que l'agriculture d'exportation et y compris dans des régions éloignées des franges périurbaines. On assiste à des investissements des citadins dans les activités rurales. Comme le relève J.-P. Raison, " affaibli d'un côté par l'exode rural sélectif, mais réanimé de l'autre par l'activité et les demandes des citadins, le monde des

12. Voir Raison J.-P., 1993, « Trente ans, trois phases de la géographie rurale dans les pays tropicaux », in L'état des savoirs sur le développement, Karthala, p. 135-159.

13. Juillard E., 1961, «L'urbanisation des campagnes », Études rurales, n 49-50, p. 5-9.

14. Mathieu N., "La notion de rural et les rapports ville-campagne en France. Des années cinquante aux années quatre-vingts », Économie rurale, $\mathrm{n}^{\circ}$ 197, p. $35-41$ (1990) ; " Rural et urbain. Unité et diversité dans les évolutions des modes d'habiter », in L'Europe et ses campagnes, Presses de Sciences Po, p. 187-216 (1996) ; et « La notion de rural et les rapports ville/campagne en France : les années quatre-vingt-dix », Économie rurale, n 247, p. 11-20, 1998 ». 15. C'est le cas par exemple du « zonage » français de l'espace qui demeure fondé sur la supériorité de la polarisation urbaine. 
campagnes est, partout sans doute, en voie de mutation rapide : changements spatiaux et changements sociaux s'y combinent dans un tableau mouvant où la dominante est sans doute le renforcement de la pression foncière et l'accroissement des inégalités» (voir note 12).

Le processus touche également l'espace « périurbain » des pays occidentaux, ce "tiers espace"(M. Vanier in Arlaud et al., 2005), lieu d'interface, de transition, à la fois urbains dans leur fonctionnement (emplois, modes de vie) et ruraux par leur "paysage» (place des usages agricoles) et par les perceptions de leurs habitants qui considèrent qu'ils vivent dans le rural. Parallèlement, bourgs et villes petites et moyennes forment l'armature des espaces ruraux et permettent aux individus qui y habitent d'accéder aux emplois et aux services.

Le débat est ouvert sur la délimitation du rural et l'émergence de "nouvelles campagnes », sur les mécanismes et processus de mobilité qui les alimentent, sur les conséquences liées aux changements d'usages, aux modifications du bâti ou aux convoitises et conflits dans le champ du foncier, des rapports sociaux ou politiques. Cette posture traverse les sciences sociales et renforce la prise en compte des stratégies de développement dans les campagnes et les « découpages pertinents » pour l'aménagement (du maillage de base aux « territoires de projet») pouvant déboucher sur des typologies renouvelées (couronnes périurbaines, espaces de faible densité, campagnes "fragiles » ou "vivantes » c'est-à-dire multifonctionnelles et autonomes).

La recherche de modèles de développement intégrés, plus durables s'impose dans les pays du Sud comme du Nord, exigeant des approches participatives et décentralisées, centrées autour des acteurs locaux et des parties prenantes (y compris urbaines). La territorialisation des pratiques et politiques publiques est mise en avant: un auteur comme N. Mathieu allant jusqu'à évoquer (voir note 1) un nouveau modèle de relation ville-campagne, véritable « contre-modèle en résistance au modèle démographique et du libéralisme économique, tout en lui empruntant (et en l'affinant) l'idée que les stratégies des individus dans leur rapport aux territoires sont essentielles. Il est aussi un retour au modèle matérialiste car, même si les réalités ont changé, il redonne un poids aux propriétés “matérielles” de tous les lieux habités, qu’ils soient ruraux ou urbains. Il est enfin éthique à nouveau parce qu'il met au centre l'individu, qu'il soit urbain ou rural, conscient que la transmission aux générations futures de territoires habitables passe par le respect de leurs valeurs écologiques, économiques et surtout sociales ». Si le rural peut être considéré comme un terrain de recherche à partir duquel peuvent être abordées les grandes questions posées aux sciences sociales, la notion de «nouvelles ruralités » intéresse les disciplines qui touchent à l'aménagement et au développement des territoires. Elle permet d'envisager les "dynamiques inscrites au cœur de nouveaux rapports villecampagne, portant à la fois sur les transformations des espaces, sur leurs usages 
résidentiels, récréatifs et productifs, sur les vécus et les représentations des acteurs, sur leur rapport à la nature, au patrimoine et aux enjeux écologiques, et sur les modes de gouvernance qui s’y déploient»(INRA, 2008).

La ruralité n'est pas un "donné » mais une construction sociale du monde, reposant sur des perceptions et des pratiques évolutives à identifier et à interpréter, devenant une clé de lecture des changements qui affectent la société toute entière. Dans leurs caractères et imaginaires, ces ruralités se recomposent rapidement. Les évolutions démographiques - entre accumulation, dépeuplement et nouvelles attractivités rurales selon les lieux -, la diversification des sociétés de plus en plus mobiles, le développement de nouveaux modes d'habiter, la réorganisation des fonctions (productives mais aussi résidentielles, de loisirs ou de "nature») et des formes de gouvernance, conduisent à une formidable diversité géographique. La question de l'éducation en zone rurale est ainsi reconfigurée et doit être revisitée à partir de recherches menées sur divers terrains et de façon interdisciplinaire.

\section{BiBLIOGRAPHIE}

ARLAUD S., JEAN Y., ROYOUX D. (2005) : Rural-Urbain. Nouveaux liens, nouvelles frontières, Presses Universitaires de Rennes, $506 \mathrm{p}$.

BONNAMOUR J. (1993) : Géographie rurale, Position et méthode, Paris, Masson, $134 \mathrm{p}$.

GUIBERT M., JEAN Y., Dynamiques des espaces ruraux dans le monde, A. Colin, 408 p. HERVIEU B., VIARD J. (2001) : Au bonheur des campagnes, Éd. de l'Aube, 126 p.

INRA (2008) : Prospective: les nouvelles ruralités en France à l'horizon 2030, Rapport, INRA, $82 \mathrm{p}$.

JOLLIVET M. et MATHIEU N. (dir.) (1989) : Du rural à l'environnement, La question de la nature aujourd'hui, L'Harmattan, $352 \mathrm{p}$.

JOLLIVET M. (2001) : Pour une science sociale à travers champs. Paysannerie, ruralité, capitalisme (France XX $X^{e}$ siècle), Éditions Arguments, $400 \mathrm{p}$.

KAYSER B., 1989, La renaissance rurale, A. Colin, 316 p.

LÉVY J., LUSSAULT M. (dir) (2003) : Dictionnaire de la Géographie et de l'espace des sociétés, Belin, $1032 \mathrm{p}$.

MENDRAS H. (1984) : La fin des paysans, Actes Sud, Avignon, rééd. 1991, 438 p. 